\title{
Sudanese diabetics with hypertension are at high risk for cardiovascular disease
}

\begin{abstract}
Objectives: To measure blood glucose, lipid profile levels, and blood pressure in diabetic hypertensive patients in order to identify the association between the parameters measured and an increased risk of cardiovascular risk in the Sudanese diabetic hypertensive patients.

Material and methods: During the months of April 2012 and March 2013, a case-control study was employed in Gezira State, Sudan. The study enrolled 200 patient who met the participation criteria, with respondents divided into diabetic hypertensive and non-diabetic categories to estimate fasting blood glucose levels (FBG), Glycosylated hemoglobin $\left(\mathrm{HbA}_{1 \mathrm{C}}\right)$ and lipid profile which include; total cholesterol (TC), high density lipoprotein cholesterol (HDL-C), low density lipoprotein cholesterol (LDL-C) and triglycerides (TG). The random access auto-analyzer bio system, A15 was used to test samples for various biochemical parameters. Individual information, as well as anthropometric and biochemical measurements were collected on a questionnaire. After each participant gave verbal consent, venous blood samples were drawn after an overnight fast. The statistical evaluation was achieved with the aid of a statistical package for social sciences (SPSS version 16, Chicago, IL, USA).

Result: The WC and BMI both increased significantly by $(\mathrm{p}=<0.0001)$, according to the analysis of variance (ANOVA). FBG and $\mathrm{HbA}_{1 \mathrm{C}}$ levels were significantly elevated by $(p=<0.0001)$. The increase in systolic blood pressure (SBP) was significant by $(p=<0.0001)$. The mean HDL-C level was at high risk (49.73) with a significant increase by $(\mathrm{p}=0.009)$. The mean LDL-C concentration was above the optimum level (109.03) with a nonsignificant increase $(\mathrm{p}=0.697)$.
\end{abstract}

Conclusion: WC, BMI, DBP, FBG, and HDL-C all increased significantly. Diabetichypertensive participants were at a high risk of develops dyslipidemia and cardiovascular disease.

Keywords: Diabetic hypertensive, lipid profile, cardiovascular disease, Sudan
Volume 9 Issue 3 - 202I

\author{
Nahla Ahmed Mohamed Abderahman,' \\ Mohamed Ahmed Ibrahim, ${ }^{2}$ Abderrhman \\ Ahmed Mohamed Ismeil, ${ }^{3}$ Nassreldeen \\ Khalid Abderahman Adam ${ }^{4}$ \\ 'Assistant professor of Biochemistry, Faculty of Medicine, \\ Department of Biochemistry, Nile Valley University, Sudan \\ ${ }^{2}$ Assistant professor of Microbiology, Faculty of Medicine, \\ Department of Microbiology, Nile Valley University, Sudan \\ ${ }^{3}$ Associate professor of Physiology, Faculty of Medicine, Sinnar \\ University, Sudan \\ 4Assistant professor of hematology, Faculty of Medical \\ Laboratory Science, University of Al Fashir, Sudan
}

Correspondence: Dr. Nahla Ahmed Mohammed Abdurrahman, Assistant professor of Biochemistry, Nile Valley University, Faculty of Medicine,Atbara, Sudan, Tel +249|23590647, Email nahlaharazawy@ymail.com

Received: May 23, 2021 | Published: June 22, 2021
Abbreviations: DM, diabetes mellitus; T2DM, type2 diabetes mellitus; HTN, hypertension; CVD, cardiovascular disease; FBG, fasting plasma glucose; HbA1C, glycosylated hemoglobin; TC, total cholesterol; TG, triglyceride; HDL-C, high density lipoprotein cholesterol; LDL-C, low density lipoprotein cholesterol; SBP, systolic blood pressure; DBP, diastolic blood pressure; WC, waist circumferance; BMI, body mass index; WHO, World health organization; p, probability; mg, milligram; $\mathrm{M}$, meter; $\mathrm{mmHg}$, millimeter of mercury

\section{Introduction}

Diabetes mellitus (DM) is one of the most prevalent chronic diseases caused by inadequate of insulin production or use. ${ }^{1}$ The most popular form of diabetes, type 2 diabetes mellitus (T2DM), is related to obesity ${ }^{2}$ which is consider as risk factor for the development of insulin resistance, hypertension (HTN) and cardiovascular diseases ${ }^{3}$ and later on increased risk of developing kidney disease. ${ }^{4}$ According to the World Health Organization (WHO), DM affects 387 million people globally and is expected to rise to 592 million adults in less than 25 years, ${ }^{5,6}$ rendering it the world's seventh leading cause of death by $2030 .{ }^{7}$ The lowest prevalence of DM was found in low-income countries, while the highest prevalence was found in upper-middleincome countries for both sexes. ${ }^{8}$ Though $80 \%$ of diabetic death was in low- and middle-income countries. ${ }^{7}$ Among all WHO's regions, the
African Region is expected to have the largest proportional increase $90.5 \%$ in the number of adult with diabetics by $2030,{ }^{7}$ it affected 14 million individuals in 2011 and this is expected to rise to about 28 million by $2030 .{ }^{9} \mathrm{DM}$ will cause five million deaths per year ${ }^{5}$ in people under the age of $60 .{ }^{10}$ Sudan is a member of the WHO's East Mediterranean region and was classified as a lower middle income country by the World Bank Income Groups in $2013^{11}$ and it has a medium prevalence of T2DM among populations. ${ }^{12}$

An epidemic of T2DM is underway in both developed and developing countries ${ }^{13}$ increased as a consequence of childhood obesity and more generally as one's age reached 40years. ${ }^{14}$ Although T2DM can be prevented by engaging in thirty minutes of moderateintensity physical exercise on most days and eating a healthy diet, inadequate access to health care and appropriate drugs, along with a lack of knowledge about diabetes, can result in complications such as blindness, amputation, cardiovascular disease (CVD), and kidney failure. ${ }^{8}$ The risk of DM complications increases with age, DM duration, insulin use, ischemic heart disease, and elevated serum creatinine. ${ }^{15}$

Hypertension is described as a persistent increase in resting arterial blood pressure (BP) of more than $140 \mathrm{~mm} \mathrm{Hg}$ systolic (SBP) and/or $90 \mathrm{~mm} \mathrm{Hg}$ diastolic (DBP). ${ }^{16}$ Age, weight, smoking habits, physical activity and a family history of (CVD) are risk factors for HTN. ${ }^{17}$ 
HTN is a progressive CV syndrome with functional and structural cardiac and vascular abnormalities that affect the heart, kidneys, brain, vasculature, and other organs, resulting in premature morbidity and death. ${ }^{18}$

In diabetic patients, high blood pressure is a risk factor for heart disease and it rises with the incidence of nephropathy, ${ }^{19}$ the existence of both nephropathy and HTN has a significant impact on heart damage in diabetics. ${ }^{20}$ Only one-third of diabetic patients achieve the recommended target blood pressure value of less than $130 / 80 \mathrm{~mm}$ Hg. ${ }^{21}$ HTN affects about one billion people worldwide, with twothirds of them living in developed countries. In 2025, 56 billion adults will have HTN, with annual deaths above 8 million and 3.2 million HTN complications. ${ }^{22}$

Glucose intolerance, T2DM, atherogenic dyslipidemia, CVD, elevated blood pressure HTN and central obesity is all representative of the metabolic syndrome. Dyslipidemia is a lipid metabolism disease that causes changes in circulating lipids and lipoprotein levels in the blood. ${ }^{23}$ This is reflected in increased TG and LDL-C, as well as a decrease in HDL-C levels. ${ }^{24}$

Such abnormalities occur in the same individual and result in several of the risk factors that frequently coexist. ${ }^{25}$ The involvement of three or more of the metabolic abnormalities mentioned below meets the criterion for metabolic syndrome: abdominal obesity or waist circumference (WC) $>102 \mathrm{~cm}$ in men and $\mathrm{WC}>88 \mathrm{~cm}$ in women, hypertriglyceridemia, TG $\geq 150 \mathrm{mg} / \mathrm{dL}$, low HDL-C levels $<40 \mathrm{mg} / \mathrm{dL}$ in men and $<50 \mathrm{mg} / \mathrm{dL}$ in women, raised blood pressure (SBP $\geq 130 \mathrm{mmHg}, \mathrm{DBP} \geq 85 \mathrm{mmHg}$ ), and raised FBG $\geq 110 \mathrm{mg} / \mathrm{dL} .{ }^{26} \mathrm{The}$ normal cholesterol content of lipoprotein is $60-70 \%$ of LDL-C, 20 $30 \%$ of HDL-C and $10-15 \%$ of VLDL of the total serum cholesterol. ${ }^{24}$

\section{Material and methods}

Study design, subject, and area: Participants were drawn from rural and urban areas in and around Wad Madani for a case-control study. The study ranged from April 2012 to March 2013. A total of 200 individuals of both males and females were enrolled in this study. One hundred diabetic hypertensive participants and 100 apparently healthy participants were participated as part of a non-diabetic or (control) group.
Inclusion criteria: The study participants ranged in age from 24 to 65 and were healthy. A total of 100 people were diagnosed as diabetic hypertensive and were administered hypoglycemic, antihypertensive, and other medications. The remaining 100 respondents were categorized as non-diabetic and seemed to be in good health

Ethical approval: The study was permitted by the State Ministry of Health's Ethics Committee.

Study method: Verbal approval was obtained. Both patients and non-diabetic participants provided bio data and anthropometric measurements; (weight was measured in kilogram (kg) and heights in meter $(\mathrm{m})$ and then the body mass index (BMI) was calculated applying the formula: $\mathrm{BMI}=($ weight in $\mathrm{kg}) /(\text { height in } \mathrm{m})^{2}(\mathrm{Ng} \mathrm{M}$, 2014).

Using the A15, a random access auto-analyzer bio system, plasma samples were examined for various biochemical parameters.

Statistical analysis: The statistical analysis was implemented using a statistical package for social sciences (SPSS version 16, Chicago, IL, USA). To compare differences in the means of continuous variables between the studies groups, analysis of variance were used (ANOVA). The frequency of the relationship between two numerical variables was measured using Chi-Squire test. P-values of 0.05 or less $(p<0.05)$ were deemed meaningful.

\section{Results}

Table 1 shows the characteristics and distribution of study group participants based on study variables. There were 100 diabetic hypertensive $(50 \%)$ and 100 non-diabetic participants in the study (non- diabetic). Females made up $148 \%$ of the study participants $(74.0 \%)$, while males made up $52(26.0 \%)$. 123(61.5\%) have moderate physical activity. When the study was taken, $88 \%$ of diabetic hypertensive patients had elevated blood pressure, while only $12 \%$ had normal blood pressure. One hundred forty-three patients $(71.5 \%)$ and $135(67.5 \%)$ had a family history of hypertension and diabetes mellitus respectively. $87(43.5 \%)$ use Antihypertensive +Hypoglycemic drug and only $2(1.0 \%)$ use dietary restriction for regulating blood glucose and pressure, and $43(43 \%)$ use additional drugs like dietary supplements and sulpho- salicylic acid. " $(\mathrm{p} \leq 0.0001),(\mathrm{p} \geq 0.0001)$.

Table I Characteristic and distribution of participants in study groups according to study variable

\begin{tabular}{|c|c|c|c|c|}
\hline Variables & & Frequency & Percent \% & Total \\
\hline \multirow[t]{2}{*}{ Group } & Diabetic hypertensive & 100 & 50 & 200 \\
\hline & non-diabetic & 100 & 50 & \\
\hline \multirow[t]{3}{*}{ Gender } & Male & 52 & 26 & 200 \\
\hline & Female & 148 & 74 & \\
\hline & Low & 54 & 27 & 200 \\
\hline \multirow[t]{2}{*}{ Physical activity } & Moderate & 123 & 61.5 & \\
\hline & High & 23 & II.5 & \\
\hline \multirow[t]{4}{*}{ HTN } & Normal (SBP\DBP= less than $\mid 20180)$ & 12 & 12 & \\
\hline & Pre-hypertension (SBP\DBP=|20 - |39|80-89) & 51 & 51 & \\
\hline & Stage I HT (SBPIDBP=|40-I59|DBP 90 -99) & 24 & 24 & 100 \\
\hline & Stage $2 \mathrm{HT}$ (SBPIDBP= 60\100 and above) & 13 & 13 & \\
\hline \multirow[t]{2}{*}{ Family History of HTN } & Yes & 143 & 71.5 & 200 \\
\hline & No & 57 & 28.5 & \\
\hline
\end{tabular}




\begin{tabular}{|c|c|c|c|c|}
\hline Variables & & Frequency & Percent \% & Total \\
\hline \multirow[t]{2}{*}{ Family History of DM } & Yes & 135 & 67.5 & 200 \\
\hline & No & 65 & 32.5 & \\
\hline \multirow[t]{4}{*}{ Medication for DM \& HTN } & Hypoglycemic Drug only & 9 & 9 & 100 \\
\hline & Dietary Control & 2 & 2 & \\
\hline & Antihypertensive + Hypoglycemic Drug & 87 & 87 & \\
\hline & Anti-Hypertensive Drug only & 2 & 2 & \\
\hline \multirow[t]{2}{*}{ Additional Drugs } & Yes & 43 & 43 & 100 \\
\hline & No & 57 & 57 & \\
\hline \multirow[t]{2}{*}{ Dietary Restriction } & Yes & 41 & 20.5 & 200 \\
\hline & No & 159 & 79.5 & \\
\hline
\end{tabular}

\section{DM, diabetes mellitus; HTN, hypertension}

Analysis of variance showed that the WC and BMI mean was (31.65) and (104.14) and they increased significantly all by $(\mathrm{p} \geq 0.0001)$ pointed to that the participants were obese and had visceral obesity. The mean concentrations of FBG was (164.63) and $\mathrm{HbA}_{1 \mathrm{C}}$ was (7.42) with highly significant by $(\mathrm{p} \geq 0.0001)$ for both indicating high $\mathrm{FBG}$ with good $\mathrm{HbA}_{1 \mathrm{C}}$. SBP mean was (128.10) with highly significant by $(\mathrm{p} \geq 0.0001)$ indicating elevated blood pressure. DBP mean was (81.40) with non-significant increase by $(p=0.782)$ because the mean of DPB of non-diabetic group was higher (82.00) than the diabetic hypertensive group. Regarding lipid profile, analysis of variance showed that; TC had non-significant increase in their mean concentrations $(189.78)$ by $(\mathrm{p}=0.220)$. HDL-C mean showed high risk (49.73) with significant increased by $(\mathrm{p}=0.009)$. LDL-C mean showed above optimal concentration level (109.03) with nonsignificant increased by (0.697). TG mean concentration was (161.57) with significant non- increased by $(\mathrm{p}=0.137)$.

\section{Discussion}

Diabetic hypertensive participants were found to be older compared with non-diabetic group with strong statistical significance (Table 2). The incidence of HTN in T2DM patients rises by $40 \%$ to $60 \%$ between the ages of 45 and 75 , indicating that HTN in diabetic patients rises with age. This conclusion is consistent with that of others. ${ }^{27}$ Participants with diabetes and hypertension had significantly higher
WC, BMI, modest physical activity, and low HDL-C concentrations. These findings were in line with those of Danquah, et al., ${ }^{28}$ and Ljungman, et al., ${ }^{29}$ who discovered that T2DM is a socioeconomic illness that mostly affects obese people and is linked to an elevated risk of HTN and hyperlipidemia in both sexes in the context of high blood pressure, BMI and family history of HTN. Diabetic hypertensive individuals showed high FBG, suggesting that the study participants had uncontrolled plasma glucose and marked rise in $\mathrm{HbA}_{1 \mathrm{C}}$, suggesting that diabetic participants were unable to meet the HbAlc target, putting them at a high risk of developing diabetes complications. Table 1 shows that $135(67.5 \%)$ of study participants had a family history of diabetes, whereas $143(71.5 \%)$ had a family history of HTN, indicating that DM and HTN are hereditary disorders. These findings were similar to those of Lillioja, et al., ${ }^{30}$; Mannino, et al., ${ }^{17}$

Dietary management, in addition to regular exercise, is the initial intervention in diabetes therapy, however in our current study food restriction had no statistical significance in the current trial due to a lack of attention to dietary plans by both the diabetes care center and the patients, but Lettieri-Barbato et al., ${ }^{31}$ showed that dietary restriction is effective in reducing adipose mass and central or visceral adiposity by diminished the biomarkers of inflammation, and increase insulin resistance.

Table 2 Analysis of variance (ANOVA)

\begin{tabular}{|c|c|c|c|c|c|c|c|}
\hline Variable & Group & Mean & Std. Deviation & Std. Error & Minimum & Maximum & P-Value \\
\hline \multirow[t]{2}{*}{ Age (years) } & Diabetic Hypertensive & 56.17 & 7.213 & 0.721 & 40 & 65 & $<0.0001$ \\
\hline & non-diabetic & 46.74 & 7.77 & 0.78 & 24 & 65 & \\
\hline \multirow[t]{2}{*}{ Weight (kg) } & Diabetic Hypertensive & 80.28 & 14.19 & 1.42 & 51 & 120 & $<0.0001$ \\
\hline & non-diabetic & 72.51 & 13.76 & 1.38 & 40 & 110 & \\
\hline \multirow[t]{2}{*}{ Height (m) } & Diabetic Hypertensive & 1.6 & 0.079 & 0.008 & 1.46 & 1.82 & 0.017 \\
\hline & non-diabetic & 1.63 & 0.1 & 0.01 & I. 4 & 1.9 & \\
\hline \multirow[t]{2}{*}{ WC (cm) } & Diabetic Hypertensive & 104.14 & 10.95 & I.I & 80 & 130 & $<0.0001$ \\
\hline & non-diabetic & 98.15 & 10.73 & 1.073 & 69 & 127 & \\
\hline \multirow[t]{2}{*}{ BMI $\left(\mathrm{kg} / \mathrm{m}^{2}\right)$} & Diabetic Hypertensive & 31.65 & 5.81 & 0.59 & 20.2 & 46.62 & $<0.0001$ \\
\hline & non-diabetic & 27.54 & 5.48 & 0.55 & 17.31 & 42.86 & \\
\hline
\end{tabular}




\begin{tabular}{|c|c|c|c|c|c|c|c|}
\hline Variable & Group & Mean & Std. Deviation & Std. Error & Minimum & Maximum & P-Value \\
\hline \multirow[t]{2}{*}{ SBP $(\mathrm{mmHg})$} & Diabetic Hypertensive & 128.1 & 14.33 & 1.43 & 100 & 180 & $<0.0001$ \\
\hline & non-diabetic & 114.3 & 13.2 & 1.32 & 80 & 170 & \\
\hline \multirow[t]{2}{*}{ DBP (mmHg) } & Diabetic Hypertensive & 81.4 & 9.32 & 0.93 & 60 & 110 & 0.782 \\
\hline & non-diabetic & 82 & 19.54 & 1.954 & 30 & 130 & \\
\hline \multirow[t]{2}{*}{ FBG (mg/dL) } & Diabetic Hypertensive & 164.63 & 66.51 & 6.65 & 57 & 380 & $<0.0001$ \\
\hline & non-diabetic & 89.77 & 24.01 & 2.401 & 46 & 242 & \\
\hline \multirow[t]{2}{*}{ HbAIC (\%) } & Diabetic Hypertensive & 7.42 & 2.08 & 0.21 & 3.4 & 12.8 & $<0.0001$ \\
\hline & non-diabetic & 5.34 & 0.76 & 0.08 & 3.45 & 6.98 & \\
\hline \multirow[t]{2}{*}{$\mathrm{TC}(\mathrm{mg} / \mathrm{dL})$} & Diabetic Hypertensive & 189.78 & 44.08 & 4.41 & 82 & 361 & 0.22 \\
\hline & non-diabetic & 197.51 & 44.85 & 4.49 & 75 & 312 & \\
\hline \multirow[t]{2}{*}{ LDL-C(mg/dL) } & Diabetic Hypertensive & 109.03 & 29.47 & 2.95 & 40 & 202 & 0.697 \\
\hline & non-diabetic & 107.36 & 31.02 & 3.1 & 32 & 184 & \\
\hline \multirow[t]{2}{*}{ HDL-C (mg/dL) } & Diabetic Hypertensive & 49.73 & 14.2 & 1.42 & 20 & 88 & 0.009 \\
\hline & non-diabetic & 55.51 & 16.89 & 1.69 & 25 & 97 & \\
\hline TG & Diabetic Hypertensive & 161.57 & 73.67 & 7.37 & 60 & 508 & 0.137 \\
\hline$(\mathrm{mg} / \mathrm{dL})$ & non-diabetic & 145.72 & 76.27 & 7.63 & 46 & 376 & \\
\hline
\end{tabular}

WC, waist circumferance; BMI, body mass index; M, meter; cm, centimeter; SBP, systolic blood pressure; DBP, diastolic blood pressure; mmHg, millimeter of mercury; FBG, fasting plasma glucose; $\mathrm{HbA}_{\mathrm{IC}}$, glycosylated hemoglobin;TC, total cholesterol;TG, triglyceride; HDL-C, high density lipoprotein cholesterol; LDL-C, low density lipoprotein cholesterol; mg, milligram; p, probability

The lipid profile showed a significant decrease in the mean concentration of HDL-C, according to Table 2 analysis of variance. The mean concentrations of TG and LDL-C both increased slightly. These findings matched those of Muna, ${ }^{32}$ who found that T2DM patients show metabolic abnormalities in both lipoprotein quality and quantity. The success of HTN medicine in the treatment of HTN, heart failure, and other cardiovascular disorders may be explained by the non-significant change in lipid profile concentrations. Our findings matched those of Otamere, et al. ${ }^{33}$ who found that participants under care had no change in lipid profile concentrations, indicating that the low level of HDL-C in this study, despite the use of anti-hypertensive drugs, suggests a high risk of improving dyslipidemia and macrovascular disease.

\section{Recommendations}

Effective diabetes treatment in Sudan demands effective diabetic care centers, properly educated staff, and strict adherence to therapy and diet; as a consequence, it is recommended to engage in personnel training, model care center creation, and patient education.

\section{Acknowledgments}

We would like to provide special thank to all participants, especially diabetic patients for sharing us this study.

\section{Funding}

None.

\section{Conflicts of interest}

The authors declare no conflict of interest.

\section{References}

1. Evans J, Newton R, Ruta D, et al. Socio-economic status, obesity and prevalence of Type 1 and Type 2 diabetes mellitus. Diabet Med 2000;17(6):478-480.

2. Jung S, Park H, Kim K, et al. Effect of weight loss on some serum cytokines in human obesity:increase in IL-10 after weight loss. $J$ Nutr Biochem. 2008;19(6):371-375.

3. Calle E, Thun M, Petrelli J, et al. Body-mass index and mortality in a prospective cohort of U.S. adults. N Engl J Med. 1999;341(15):10971105

4. Ritz E. Nephropathy in type 2 diabetes. J Intern Med. 1999;245(2):111126.

5. Zinman B. The International diabetes Federation World diabetes congress 2015. Eur Endocrinol. 2015;11(2):66.

6. Mathers C, Loncar D. Projections of global mortality and burden of disease from 2002 to 2030. PLoS Med. 2006;3(11):442.

7. Whiting D, Guariguata L, Weil C, et al. IDF diabetes atlas:global estimates of the prevalence of diabetes for 2011 and 2030. Diabetes Res Clin Pract. 2011;94 (3):311-321.

8. Islam S, Purnat T, Phuong N, et al. Non-communicable diseases (NCDs) in developing countries:a symposium report. Global Health. 2014;10:81.

9. L'Heveder R, Nolan T. International Diabetes Federation. Diabetes Res Clin Pract. 2013;101(3):349-351.

10. Bloomgarden Z. Questioning glucose measurements used in the International diabetes Federation (IDF) Atlas. J Diabetes. 2016;8 (6):746747.

11. Boutayeb A, Boutayeb W, Maamri A, et al. The rise of diabetes prevalence in the Arab region. Open J Epidemiology. 2012;2:55-60. 
12. Elbagir M, Eltom M, Elmahadi E, et al. A high prevalence of diabetes mellitus and impaired glucose tolerance in the Danagla community in northern Sudan. Diabet Med. 1998;15(2):164-169.

13. Kronenberg HM, Melmed S, Polonsky KS, et al. Williams textbook of endocrinology. 11th edn. Saunders; 2008:1329-1563.

14. Colin D, Gareth W, David A, et al. Disorders of glucose homeostasis. Oxford Textbook of Medicine. 5th edn. New York: Oxford; 2003.

15. Nichols G, Hillier T, Erbey J, et al. Congestive heart failure in type 2 diabetes:prevalence, incidence, and risk factors. Diabetes Care. 2001;24(9):1614-1619.

16. Anderssen S, Holme I, Urdal P, et al. Diet and exercise intervention have favourable effects on blood pressure in mild hypertensives:the Oslo Diet and Exercise Study (ODES (Blood Press. 1995;4(6):343-349.

17. Mannino D, Caraballo R, Benowitz N, et al. Predictors of cotinine levels in US children:data from the Third National Health and Nutrition Examination Survey. Chest. 2001;120(3):718-724.

18. Giles T, Berk B, Black H, et al. Expanding the definition and classification of hypertension. J Clin Hypertens (Greenwich). 2005;7(9):505-512.

19. Tarnow L, Rossing P, Gall M, et al. Prevalence of arterial hypertension in diabetic patients before and after the JNC-V. Diabetes Care. 1994;17(11):1247-1251.

20. Van Hoeven K, Factor S. A comparison of the pathological spectrum of hypertensive, diabetic, and hypertensive-diabetic heart disease. Circulation. 1990;82(3):848-855.

21. Leenen F, Dumais J, McInnis N, et al. Results of the Ontario survey on the prevalence and control of hypertension. CMAJ. 2008;178(11):1441-1449.

22. Atlas D. Hypertenion International Diabetes Federation. 2nd edition. 2003.

23. Giuliano I, Caramelli B. Dis-lipidemiasnainfância e adolescência. Pediatria. 2008;29:275-285.

24. Expert Panel on Detection and Treatment of High Blood Cholesterol in Executive Summary of The Third Report of The National Cholesterol Education Program (NCEP) Expert Panel on Detection, Evaluation, And Treatment of High Blood Cholesterol In Adults (Adult Treatment Panel III)(2001). JAMA 285 (19):2486-2497.
25. Sattar N, Wannamethee G, Sarwar N, et al. Metabolic syndrome with and without $\mathrm{C}$-reactive protein as a predictor of coronary heart disease and diabetes in the West of Scotland Coronary Prevention Study. Circulation. 2003;108(4):414-419.

26. Matthews D, Hosker J, Rudenski A, et al. Homeostasis model assessment:insulin resistance and beta-cell function from fasting plasma glucose and insulin concentrations in man. Diabetologia. 1985;28(7):412419.

27. Harris M, Cowie C, Stern M, et al. Diabetes in America. Washington, DC: National Institutes of Health, National Institute of Diabetes and Digestive and Kidney Diseases. 2nd edn. National Institute of Diabetes and Digestive and Kidney Diseases; 1995.

28. Danquah I, Bedu-Addo G, Terpe K, et al. Diabetes mellitus type 2 in urban Ghana:characteristics and associated factors. BMC Public Health. 2012.

29. Ljungman S, Wikstrand J, Hartford M, et al. Urinary albumin excretion-a predictor of risk of cardiovascular disease. A prospective 10-year follow-up of middle-aged nondiabetic normal and hypertensive men. Am J Hypertens. 1996;9(8):770-778.

30. Lillioja S, Mott D, Spraul M, et al. Insulin resistance and insulin secretory dysfunction as precursors of non-insulin-dependent diabetes mellitus. Prospective studies of Pima Indians. N Engl J Med. 1993;329(27):19881992.

31. Lettieri-Barbato D, Giovannetti E, Aquilano K. Effects of dietary restriction on adipose mass and biomarkers of healthy aging in human. Aging (Albany NY). 2016;8(12):3341-3355.

32. Muna W. Cardiovascular disorders in Africa. World Health Stat Q. 1993;46(2):125-133.

33. Otamere H, Aloamaka C, Okokhere P, et al. Lipid Profile in Diabetes Mellitus; What Impact Has Age and Duration. British Journal of Pharmacology and Toxicology. 2011;2(3):135-137. 